\title{
NULL SETS AND ESSENTIALLY SMOOTH LIPSCHITZ FUNCTIONS*
}

\author{
JONATHAN M. BORWEIN ${ }^{\dagger}$ AND WARREN B. MOORS ${ }^{\ddagger}$
}

\begin{abstract}
In this paper we extend the notion of a Lebesgue-null set to a notion which is valid in any completely metrizable Abelian topological group. We then use this definition to introduce and study the class of essentially smooth functions. These are, roughly speaking, those Lipschitz functions which are smooth (in each direction) almost everywhere.
\end{abstract}

Key words. Lipschitz functions, Haar-null sets, essentially smooth functions

AMS subject classifications. Primary, 49J520, 46N10; Secondary, 58C20

PII. S1052623496305213

1. Introduction. The primary purpose of this paper is to introduce a new class of Lipschitz functions and present some of their most fundamental properties. In order to accomplish this goal, we must first extend the notion of a Lebesgue-null set to a notion which is valid in any Banach space. In the case of separable Banach spaces this task has already been achieved. In fact, for separable Banach spaces, there have been several successful solutions to this problem (see [20, 15, 7], to name just a few). For our purposes the most useful generalization of a "null" set is that of the Haar-null (or zero) set introduced by J. P. R. Christensen in [7]. Indeed, in section 2 of this paper we retrace and extend the results of Christensen to spaces which are not necessarily separable. While some of our extensions need additional work, many follow naturally from their separable counterparts.

In section 3 we introduce the class of "essentially smooth" Lipschitz functions. Loosely speaking, these are those functions which are smooth (in each direction) almost everywhere, that is, smooth (in each direction) everywhere except on a "null" set. As we shall come to see, the essentially smooth functions enjoy particularly desirable differentiability properties. For instance, they are all integrable and $D$ representable (on $\operatorname{class}(\mathcal{S})$ spaces) in the sense defined within.

This class of functions is of central importance in nonsmooth optimization. The importance of the class stems (see $[2,4,5,6]$ ) from the fact that it provably provides the largest robust class of functions

- stable under the most significant operations of calculus and of formation of marginal functions [4];

- uniquely determined by their Clarke subgradients [4];

- smooth enough for algorithmic purposes [4, 18];

- possessing strong chain rules [5].

A primary utility of this paper is to provide coherent tools for the use of "measuretheoretic" techniques in nonseparable spaces, such as $\mathrm{E}^{\infty}$, which are frequently natural settings for optimization and control problems.

\footnotetext{
* Received by the editors June 12, 1996; accepted for publication (in revised form) January 21, 1997.

http://www.siam.org/journals/siopt/8-2/30521.html

${ }^{\dagger}$ CECM, Department of Mathematics and Statistics, Simon Fraser University, Burnaby, BC V5A 1S6, Canada (jborwein@cecm.sfu.ca). This author was supported by NSERC and the Shrum Endowment at Simon Fraser University.

$¥$ Department of Mathematics, The University of Auckland, Private Bag 92019, Auckland, New Zealand (moors@math.auckland.ac.nz). This author was supported by a New Zealand Science and Technology Post-Doctoral Fellowship.
} 
2. Haar-null sets. Throughout this section of the paper, $(G,+, \tau)$ will denote a completely metrizable Abelian topological group; that is, $(G, \tau)$ is homeomorphic to a complete metric space. Due to a result of V. Klee (see [14]), we may and do assume that the topology $\tau$ is generated by a metric $d$, which is both complete and invariant.

In our terminology a measure will always be nonnegative and not identical to zero. By a Borel measure on a topological space $X$ we mean any measure defined on $\mathcal{B}(X)$ - the Borel subsets of $X$. By a Radon measure $\mu$ on $X$ we mean any Borel measure on $X$ extended to its completion on $X$, which satisfies

(i) $\mu(K)<\infty$ for each compact subset $K \subseteq X$;

(ii) $\mu(A)=\sup \{\mu(K): K \subseteq A, K$ compact $\}$ for each $A \in \mathcal{B}(X)$.

Note that (ii) actually holds for each $A$ in the $\mu$-completion of $\mathcal{B}(X)$. In addition, if $\mu$ is finite (that is, $\mu(X)<\infty$ ), then $\mu(A)=\inf \{\mu(U): A \subseteq U, U$ open $\}$ for each $A$ in the $\mu$-completion of $\mathcal{B}(X)$.

We will say that a subset $A$ of a topological space $X$ is universally (Radon) measurable if it belongs to the $\mu$-completion of each finite (Radon) Borel measure $\mu$ on $X$, and we shall denote by $\mathcal{U}(X)\left(\mathcal{U}_{R}(X)\right)$ the family of all universally (Radon) measurable subsets on $X$. Observe that $\mathcal{B}(X) \subseteq \mathcal{U}(X) \subseteq \mathcal{U}_{R}(X)$. (In the case when $X$ is Polish, $\mathcal{U}(X)=\mathcal{U}_{R}(X)$; see Theorem 2.2.) It follows immediately from this definition that on any topological group the universally (Radon) measurable sets form a translation invariant $\sigma$-algebra.

A natural question to ask at this point is whether the family of all universally measurable subsets properly contains the Borel sets. One way to see the affirmative answer to this is the following.

Given a completely regular topological space $X$, let $\mathcal{D}(X)$ denote the smallest $\sigma$-algebra on $X$ which contains all the Borel subsets and is stable under the Souslin operation. (Let us refresh our memory on the definition of the Souslin operation. We say that a family of sets $\mathcal{A}$ is closed under the Souslin operation if all sets of the form

$$
\bigcup\left\{\bigcap\{A(\sigma \mid n): n \geq 1\}: \sigma \in N^{N}\right\}, \quad A(\sigma \mid n) \in \mathcal{A}
$$

(here we use $\sigma \mid n$ to denote $\sigma_{1}, \sigma_{2}, \ldots, \sigma_{n}$ when $\left(\sigma_{1}, \sigma_{2}, \ldots, \sigma_{n}, \ldots\right) \in N^{N}$ )

are contained in $\mathcal{A}$.) By using the fact that for each complete finite measure $\mu$ on $X$ the family of all $\mu$-measurable sets is closed under the Souslin operation, we may deduce that each member of $\mathcal{D}(X)$ is universally measurable (see [21]). Now, it is known that $\mathcal{D}(X)$ contains all the Cech-analytic subsets of $X$, which, in turn, contain all the analytic subsets of $X$ (see [12]). (Recall that a subset $A \subseteq X$ is analytic if it is the continuous image of a complete, separable metric space; that is, the continuous image of a Polish space.) Finally, it is also known that each uncountable Polish space contains an analytic set which is not a Borel set (see [13, p. 201]).

We are now in a position to define our "null" sets. Let $(G,+, \tau)$ be a completely metrizable Abelian topological group. We will call a universally Radon measurable set $A \subseteq G$ a Haar-null set if there exists a (not necessarily unique) Radon probability measure $p$ on $G$ such that $p(g+A)=0$ for each $g \in G$. (In such a case, we shall call the measure $p$ a test measure for $A$.) More generally, we will say that a subset $A \subseteq G$ is a Haar-null set if it is contained in a universally Radon measurable Haar-null set. It will sometimes be useful to remember that if $p$ is a test measure for $A$, then $g+A$ is $p$-measurable for each $g \in G$, even though the set $A$ may not be universally Radon measurable. We should also note that the Dirac (point-mass) measures are rarely useful as test measures since the only set they can test is the empty set. 
In order to simplify the later part of this section, we will take this opportunity to present some of the fundamental properties possessed by Radon measures. All of the results presented in the next theorem are either straightforward or may be found (more or less as stated) in Chapter 2 of [1].

THEOREM 2.1. Let $X$ and $Y$ be Hausdorff topological spaces, and let $Z$ be a Borel subset of $X$. Further, let $\mu$ be a Radon measure on $X$ and $\nu$ be a Radon measure on $Y$. Then we have the following:

(a) The space $X$ contains a smallest closed subset $A \subseteq X$ (called the support of $\mu$ ) such that $\mu(X \backslash A)=0$. It follows immediately from this definition that, for each open subset $U \subseteq X, \mu(U \cap A)>0$ whenever $U \cap A \neq 0$. Furthermore, if $X$ is metrizable and $\mu(X)<\infty$, then the support of $\mu$ is separable.

(b) If we define $\mu_{Z}: \mathcal{B}(Z) \rightarrow[0, \infty]$ by $\mu_{Z}(A) \equiv \mu(A)$ for each $A \in \mathcal{B}(Z)$, then (the completion of) $\mu_{Z}$ defines a Radon measure on $Z$, which we will call the restriction of $\mu$.

(c) Conversely, if we are given a finite Radon measure $\mu_{Z}$ on $Z$ and we define $\eta: \mathcal{B}(X) \rightarrow[0, \infty]$ by $\eta(A) \equiv \mu_{Z}(A \cap Z)$ for each $A \in \mathcal{B}(X)$ then (the completion of) $\eta$ is a Radon measure on $X$, which in the future we will call the extension of $\mu_{Z}$. (Note that if $Z$ is a closed subset of $X$, then we need not assume that $\mu_{Z}$ is finite.)

(d) $\mathcal{U}_{R}(Z)=\left\{A \in \mathcal{U}_{R}(X): A \subseteq Z\right\}$

(e) If $\mu$ is $\sigma$-finite, that is, $X=\bigcup\left\{X_{n}: n \in N\right\}$ where each $X_{n} \in \mathcal{B}(X)$ and $\mu\left(X_{n}\right)<\infty$, then each member of $\mathcal{U}_{R}(X)$ is $\mu$-measurable.

(f) If $A$ is a universally Radon measurable subset of $X \times Y$ and both the measures $\mu$ and $\nu$ are $\sigma$-finite, then the mapping $x \rightarrow \nu\left([A]_{X}(x)\right)$ is universally Radon measurable on $X$ and $y \rightarrow \mu\left([A]_{Y}(y)\right)$ is universally Radon measurable on $Y$. (Here, $[A]_{X}(x) \equiv$ $\{y \in Y:(x, y) \in A\}$ and $[A]_{Y}(y) \equiv\{x \in X:(x, y) \in A\}$.) Moreover, there exists a unique Radon product measure on $X \times Y$, denoted $\mu \otimes \nu$, that restricts to the usual product measure on $\mathcal{B}(X) \times \mathcal{B}(Y)$ and for which

$$
\int_{A} d(\mu \otimes \nu)=\int_{X} \nu\left([A]_{X}(x)\right) d \mu=\int_{Y} \mu\left([A]_{Y}(y)\right) d \nu .
$$

(g) If $f$ is a continuous mapping from $X$ into $Y$ and $\mu(X)<\infty$, then the measure $\mu_{f}: \mathcal{B}(Y) \rightarrow[0, \infty]$ defined by $\mu_{f}(A) \equiv \mu\left(f^{-1}(A)\right)$ extends to define a Radon measure on $Y$. Moreover, $f^{-1}(A) \in \mathcal{U}_{R}(X)$ for each $A \in \mathcal{U}_{R}(Y)$.

(h) If $\mu$ and $\nu$ are finite measures and $X=Y$, then we may define the convolution of $\mu$ and $\nu$ as $\mu * \nu \equiv(\mu \otimes \nu)_{+}$; that is,

$$
\mu * \nu(A) \equiv \mu \otimes \nu(\{(x, y): x+y \in A\}) \text { for each } A \in \mathcal{B}(X) .
$$

The completion of this measure is a finite Radon measure on $X$.

The convolution defined above plays a central role in what follows.

Remark 2.1. It follows from (f) and (h) above that if $p_{A}$ is a test measure for a set $A$ and $p_{B}$ is a test measure for a set $B$, then $p_{A} * p_{B}$ is a test measure for $A \cup B$. Indeed, since $p_{A} * p_{B}(A \cup B) \leq p_{A} * p_{B}(A)+p_{A} * p_{B}(B)$, it suffices to observe that $p_{A} * p_{B}(A)=0$ and $p_{A} * p_{B}(B)=0$. As a consequence of this, we may deduce that the Haar-null sets are closed under finite unions. Later, we shall see that this remains true of countable unions.

Before we present the elementary properties possessed by Haar-null sets, we will digress for a moment so that we may justify calling our "null" sets Haar-null sets, as in [7]. 
THEOREM 2.2 (Ulam's theorem [9, p. 176]). Let $(X, \tau)$ be a separable and completely metrizable topological space (that is, $X$ is a Polish space). Then the completion of each finite Borel measure on $X$ defines a finite Radon measure on $X$.

A generalization of the above theorem is the following.

Remark 2.2 If the support of a finite Borel measure is Polish, then its completion is a finite Radon measure. In particular, if $(X, \tau)$ is metrizable, then each finite Borel measure on $X$, with compact support, extends to be a finite Radon measure on $X$.

So we see then that the definition of a Haar-null set given in this paper is consistent with that given in [7]. Moreover, it also coincides with the definition of a shy set, as given in [11].

A key set of facts is listed next.

Proposition 2.3. Let $(G,+, \tau)$ be a completely metrizable Abelian group. Then we have the following:

(a) Every subset of a Haar-null set is Haar-null.

(b) If $A$ is a Haar-null set, then so is $g+A$ for every $g \in G$.

(c) If $A$ is a Haar-null set, then there exists a test measure for $A$ with compact support.

(d) Suppose that $A$ is a subset of a universally Radon measurable set $B$. Then to show that $A$ is a Haar-null set, it is sufficient to show that there exists a test measure for $B$ which is $\sigma$-finite. Note that if $G$ is Polish, then all Radon measures on $G$ are $\sigma$-finite (see [1, p. 62]), and so in this case it is sufficient to use any Radon measure. If $B$ is a Borel set, then we may use any Borel measure $\mu$ (as a test measure) for which there exists a compact subset $K \subseteq G$ with $0<\mu(K)<\infty$.

(e) If $A$ is a Haar-null set, then $G \backslash A$ is dense in $G$.

(f) If $\left\{A_{j}: j \in N\right\}$ are Haar-null sets, then so is $\bigcup\left\{A_{j}: j \in N\right\}$.

(g) If $G$ is a locally compact Polish group, then a subset $A \subseteq G$ is a Haar-null set if and only if $\lambda(A)=0$ for the Haar-measure $\lambda$ on $G$.

Proof. The proof of the facts (a)-(c) are obvious and hence left to the reader.

(d) Suppose that $\mu$ is a test measure for the set $B$. Let $K$ be any compact subset of $G$ such that $0<\mu(K)<\infty$. It is easy to see that the finite Radon measure, $\mu_{K}$, defined by $\mu_{K}(X) \equiv \mu(K \cap X)$ for each $X \in \mathcal{B}(G)$ is also a test measure for the set $B$.

To prove (e) it is sufficient to show that there are no (nonempty) open Haarnull sets. To this end, let $U$ be a nonempty open subset of $G$ and suppose, to the contrary, that there does exist a test measure $p$ for $U$ on $G$. Let $A$ denote the support of $p$. It is easy to see that for some $g_{0} \in G,\left(g_{0}+U\right) \cap A \neq 0$. Hence, $p\left(g_{0}+U\right) \geq p\left(\left(g_{0}+U\right) \cap A\right)>0$, which contradicts the fact that $p$ is a test measure for $U$. Hence, no nonempty open subset of $G$ is a Haar-null set.

(f) Let us first observe that without loss of generality we may assume that each set $A_{j}$ is universally Radon measurable. For each $j \in N$, let $p_{j}$ be a test measure for $A_{j}$ on $G$. Let $H$ be the smallest closed subgroup of $G$ that contains the support of each $p_{j}$. Since the support of each $p_{j}$ is separable (see Theorem 2.1 (a)) it is not too difficult to see that $H$ is Polish. Next, let $p_{j}^{*}$ denote the restriction of $p_{j}$ to $H$. We know from Theorem 1 in [7] that there exists a Radon probability measure $p^{*}$ on $H$ that is a test measure for each set of the form $\bigcup\left\{B_{j}: j \in N\right\}$, provided that $B_{j} \in \mathcal{U}_{R}(H)$ and $p_{j}^{*}$ is a test measure for $B_{j}$. (The measure $p^{*}$ is essentially the infinite convolution of the $p_{j}^{*}$.) Let $p$ be the extension, to $G$, of $p^{*}$. We claim that $p$ is a test measure for $\bigcup\left\{A_{j}: j \in N\right\}$. To prove this, we must show that for each $g \in G$, 
$p\left(g+\bigcup\left\{A_{j}: j \in N\right\}\right)=0$. So let us fix $g \in G$. Then,

$$
p\left(g+\bigcup_{j=1}^{\infty} A_{j}\right)=p^{*}\left(\left(g+\bigcup_{j=1}^{\infty} A_{j}\right) \cap H\right)=p^{*}\left(\bigcup_{j=1}^{\infty}\left(g+A_{j}\right) \cap H\right) .
$$

However, each $p_{j}^{*}$ is a test measure for $\left(g+A_{j}\right) \cap H$ since each $p_{j}$ is a test measure for $A_{j}$, and $h+\left(\left(g+A_{j}\right) \cap H\right)=\left((h+g)+A_{j}\right) \cap H \subseteq(h+g)+A_{j}$ for each $h \in H$. Therefore, $p^{*}$ is a test measure for $\bigcup\left\{\left(g+A_{j}\right) \cap H: j \in N\right\}$, and so $p^{*}\left(\bigcup\left\{\left(g+A_{j}\right) \cap H: j \in N\right\}\right)=0$, which gives that $p\left(g+\bigcup\left\{A_{j}: j \in N\right\}\right)=0$.

Let us also note that an alternative proof of this is given in [11]. We should, however, lend a note of caution here that in their proof they implicitly assume that the metric under consideration is both translation invariant and complete. (Of course, from our remark at the start of this section, we see that such an assumption is indeed valid.)

(g) The proof of this fact may be found in [7].

One of the most powerful theorems in measure theory is Fubini's theorem, and so it is natural to want to determine whether there is a version of Fubini's theorem that holds for Haar-null sets. Disappointingly, in [7] the author gives an example to show that we must banish all hope of obtaining a full version of Fubini's theorem. Nonetheless, in this same paper the author indicates (without proof) that a weaker version of Fubini's theorem does hold (in a Polish Abelian group). We next take the opportunity to record a proof of this theorem in the setting of completely metrizable Abelian groups.

THEOREM 2.4. If $\left(H,+, \tau_{1}\right)$ is a completely metrizable Abelian group and $(T,+$, $\left.\tau_{2}\right)$ is a locally compact Polish Abelian group, then for each universally Radon measurable subset $A \subseteq H \times T$ (the product group) the following are equivalent:

(i) $[A]_{H}(h)$ is a Haar-null set, for the Haar-measure on $T$, for almost all $h \in H$.

(ii) The set $A$ is a Haar-null set in the product group $H \times T$.

Proof. Let $p_{T}$ be a Radon probability measure on $T$ that is equivalent to the Haar-measure on $T$; that is, for each $B \in \mathcal{U}_{R}(T), p_{T}(B)=0$ if and only if $B$ is a null set for the Haar-measure on $T$. (Note that such a measure $p_{T}$ exists since $T$, with the Haar-measure, is $\sigma$-finite.)

Suppose that (i) holds. Let $A_{H} \equiv\left\{h \in H: \mu\left([A]_{H}(h)\right)>0\right\}$. Let $p$ be a test measure for the set $A_{H}$. We claim that $p \otimes p_{T}$ is a test measure for $A$. Fix $\left(h_{0}, t_{0}\right) \in H \times T$. Then

$$
\left\{h \in H: p_{T}\left(\left[\left(h_{0}, t_{0}\right)+A\right]_{H}(h)\right)>0\right\}=h_{0}+A_{H} .
$$

The result now follows from Theorem 2.1 (f).

Suppose that (ii) holds. Let $\delta_{0}$ denote the Dirac (point-mass) measure at $0_{H}$ and define $\mu \equiv \delta_{0} \otimes p_{T}$. Let $p$ be a test measure for the set $A$ and set $A_{H} \equiv\{h \in H$ : $\left.p_{T}\left([A]_{H}(h)\right)>0\right\}$. We know from Theorem 2.1 (f) that $A_{H}$ is universally Radon measurable, so it suffices to construct a test measure for $A_{H}$. To this end, we define a Radon measure $p_{H}$ on $H$ by $p_{H}(B) \equiv p(B \times T)$ for each $B \in \mathcal{U}_{R}(H)$. (Note that $p_{H}(B) \equiv p\left(P^{-1}(B)\right)$, where $P$ is the natural projection of $H \times T$ onto $H$.) Fix $h_{0} \in H$ then

$$
0=\mu * p\left(\left(h_{0}, 0_{T}\right)+A\right)=\int_{H \times T} \mu\left(A+\left(h_{0}-h,-t\right)\right) d p(h, t) .
$$


Now, the mapping $(h, t) \rightarrow \mu\left(A+\left(h_{0}-h,-t\right)\right)=p_{T}\left(\left[A+\left(0_{H},-t\right)\right]_{H}\left(h-h_{0}\right)\right)$ is universally Radon measurable and hence $p$-measurable (see Theorem 2.1 (f) and (g)) and strictly positive on $\left(h_{0}+A_{H}\right) \times T$.

Hence, $p_{H}\left(h_{0}+A_{H}\right)=p\left(\left(h_{0}+A_{H}\right) \times T\right)=0$.

There is one significant problem which, up until this point, we have neglected. That is, how does one actually determine whether a given set is Haar-null? In general, this is a difficult question to answer. In some situations, however, the following facts are helpful.

Proposition 2.5. Let $A$ be a Borel subset of a completely metrizable Abelian group $(G,+, \tau)$. Then $A$ is a Haar-null set if for some locally compact subgroup $H$ of $G, \lambda((g+A) \cap H)=0$ for each $g$ in some transversal $T$ to $H$ in $G$. Here, $\lambda$ denotes the Haar-measure on $H$.

Let us recall the definition of a transversal. A subset $T$ of a group $G$, with normal subgroup $H$, which contains just one element from each coset of $H$ is called a transversal to $H$ in $G$.

The next condition is stated in [7] for Polish groups. However, we note here that the proof in no way relies on the fact that the group is separable.

Proposition 2.6. Let $A$ and $B$ be arbitrary universally Radon measurable sets in a completely metrizable Abelian group $G$. If we put

$$
F(A, B) \equiv\{g \in G:(g+A) \cap B \text { is not a Haar-null set }\},
$$

then $F(A, B)$ is an open subset of $G$ (possibly empty). In particular, if $A$ is not a Haar-null set, then $F(A, A)$ is an open neighborhood of $0_{G}$.

COROLlaRY 2.7. If $(G,+, \tau)$ is a completely metrizable Abelian group which is not locally compact, then each compact subset is Haar-null.

Proof. Suppose that, to the contrary, there exists a compact subset $K \subseteq G$ which is not a Haar-null set. Then, by Proposition 2.6, $F(K, K)$ is an open neighborhood of $0_{G}$. However, $0_{G} \in F(K, K) \subseteq K-K$, which is compact. Therefore, all compact subsets of $G$ must be Haar-null.

By employing considerably more effort, one can show, in the setting of Banach spaces, that certain more complicated sets are Haar-null. For instance, in [3] the authors show that the boundary of every closed convex subset of every separable super-reflexive Banach space is Haar-null. Moreover, it is shown in [16] that the boundary of every closed convex nowhere-dense set in any super-reflexive space is Haar-null. (Note that in [16] the author does not use the Haar-null terminology.) It is known (see [17]) that every separable nonreflexive Banach space contains a closed convex subset whose boundary is not Haar-null. (Note that such a set must have no interior.)

We end this section with an example that shows that the completeness of the group $G$ in the proof of Proposition 2.3 part (f) is indispensable.

Example 2.1. Let $(X,\|\cdot\|)$ be an infinite dimensional normed linear space such that $X=\bigcup\left\{X_{n}: n \in N\right\}$, where each $X_{n}$ is a finite dimensional subspace of $X$. It follows from Proposition 2.5 that each subspace $X_{n}$ is a Haar-null subset of $X$. (To see this, consider the following. For each $n \in N$, let $H_{n}$ be a one-dimensional subspace of $X$ such that $H_{n} \nsubseteq X_{n}$ and let $\lambda_{n}$ denote the Lebesgue measure on $H_{n}$, then $\lambda_{n}\left(\left(x+X_{n}\right) \cap H_{n}\right)=0$ for each $x \in X$.) However, $p\left(\bigcup\left\{X_{n}: n \in N\right\}\right)=1$ for each probability measure $p$ on $X$.

3. Essentially smooth Lipschitz functions. In this section of the paper we will introduce the class of essentially smooth Lipschitz functions and develop some of 
their most fundamental properties. We begin by recalling some preliminary definitions regarding the Clarke generalized directional derivative [8]. A real-valued function $f$ defined on a nonempty open subset $U$ of a Banach space $X$ is said to be locally Lipschitz on $U$ if for each $x_{0} \in U$ there exists a $K>0$ and a $\delta>0$ such that

$$
|f(x)-f(y)| \leq K\|x-y\| \quad \text { for all } \quad x, y \in B\left(x_{0}, \delta\right) \cap U .
$$

For functions in this class, we will consider the following three directional derivatives:

(i) The upper Dini derivative at $x \in U$, in the direction $y$, given by

$$
f^{+}(x ; y) \equiv \limsup _{\lambda \rightarrow 0^{+}} \frac{f(x+\lambda y)-f(x)}{\lambda} .
$$

(ii) The lower Dini derivative at $x \in U$, in the direction $y$, given by

$$
f^{-}(x ; y) \equiv \liminf _{\lambda \rightarrow 0^{+}} \frac{f(x+\lambda y)-f(x)}{\lambda} .
$$

(iii) The Clarke generalized directional derivative at $x \in U$, in the direction $y$, given by

$$
f^{0}(x ; y) \equiv \limsup _{\substack{z \rightarrow x \\ \lambda \rightarrow 0^{+}}} \frac{f(z+\lambda y)-f(z)}{\lambda} .
$$

It is immediate from these definitions that for each $x \in U$ and each $y \in X$

$$
f^{-}(x ; y) \leq f^{+}(x ; y) \leq f^{0}(x ; y) .
$$

An initially surprising but important fact regarding the Clarke generalized directional derivative is that for each $x_{0} \in U$, the mapping $y \rightarrow f^{0}\left(x_{0} ; y\right)$ is a continuous sublinear functional on $X$ (see [8]). Associated with the Clarke generalized directional derivative is the Clarke subdifferential mapping, which is defined by

$$
\partial f(x) \equiv\left\{x^{*} \in X^{*}: x^{*}(y) \leq f^{0}(x ; y) \text { for each } y \in X\right\} \text { for each } x \in U .
$$

Next, we shall examine some notions of differentiability. We say that a real-valued locally Lipschitz function $f$ is differentiable at $x$, in the direction $y$, if

$$
f^{\prime}(x ; y) \equiv \lim _{\lambda \rightarrow 0} \frac{f(x+\lambda y)-f(x)}{\lambda} \quad \text { exists }
$$

and we say that $f$ is Gâteaux differentiable at $x$ if

$$
\nabla f(x)(y) \equiv \lim _{\lambda \rightarrow 0} \frac{f(x+\lambda y)-f(x)}{\lambda} \quad \text { exists }
$$

for each $y \in X$ and $\nabla f(x)$ is a continuous linear functional on $X$.

We shall also be interested in a slightly stronger notion of differentiability. A locally Lipschitz function $f$ is said to be strictly differentiable at $x$, in the direction $y$, if

$$
\lim _{\substack{z \rightarrow x \\ \lambda \rightarrow 0^{+}}} \frac{f(z+\lambda y)-f(z)}{\lambda} \text { exists, }
$$


and we say that $f$ is strictly differentiable at $x$, if $f$ is strictly differentiable at $x$, in every direction $y \in X$. We recall that a locally Lipschitz function $f$ is strictly differentiable at $x$, in the direction $y$, if and only if

$$
f^{0}(x ; y)=f^{\prime}(x ; y)=-f^{0}(x ;-y) .
$$

Our first theorem in this section shows us that the Clarke generalized directional derivative is insensitive to Haar-null sets.

LEMMA 3.1 (Mean-Value theorem). Let $f$ be a real-valued locally Lipschitz function defined on a nonempty open subset of the real line, which contains the nondegenerate interval $[a, b]$. Then there exists a Borel subset $M$ of $[a, b]$, with positive measure, such that for each $t \in M, f^{\prime}(t)$ exists and

$$
f^{\prime}(t) \geq \frac{f(b)-f(a)}{b-a} .
$$

THEOREM 3.2. Let $f$ be a real-valued locally Lipschitz function defined on a nonempty open subset $U$ of a Banach space $X$. Then for each $x \in U, y \in X \backslash\{0\}$, and Haar-null set $N \subseteq X$, we have that

$$
f^{0}(x ; y)=\limsup _{\substack{z \rightarrow x \\ z \in U \backslash N}} f^{-}(z ; y) \quad-f^{0}(x ;-y)=\liminf _{\substack{z \rightarrow x \\ z \in U \backslash N}} f^{+}(z ; y) .
$$

Proof. Since the proof of the second case is identical to that of the first, we shall only consider the first case. Fix $x \in U, y \in X \backslash\{0\}$, and let $N$ be a Haar-null subset of $X$. (Note that, by possibly making $N$ bigger, we may assume that it is universally Radon measurable.) We begin by observing that

$$
\limsup _{\substack{z \rightarrow x \\ z \in U \backslash N}} f^{-}(z ; y) \leq \limsup _{z \rightarrow x} f^{+}(z ; y)=f^{0}(x ; y) .
$$

So it is sufficient to show that

$$
f^{0}(x ; y) \leq \limsup _{\substack{z \rightarrow x \\ z \in U \backslash N}} f^{-}(z ; y) .
$$

Indeed, to accomplish this, it will be sufficient to show that for each $\epsilon>0$ and each $\delta>0$

$$
f^{0}(x ; y)-\epsilon<\sup \left\{f^{-}(z ; y):\|z-x\| \leq \delta \text { and } z \in U \backslash N\right\} .
$$

So suppose $\epsilon>0$ and $\delta>0$ are given. Note that, by possibly making $\delta$ smaller, we may assume that $B(x, \delta) \subseteq U$. Let $H$ be any closed hyperplane in $X$ that does not contain the point $y$, and consider the isomorphism $T: H \times R \rightarrow X$ defined by $T(h, r) \equiv h+r y$. If we set $N_{H} \equiv\{h \in H: \lambda(\{r \in R: T(h, r) \in N\})>0\}$, then by Theorem 2.4 we have that $N_{H}$ is a Haar-null subset of $H$.

Now, by the definition of $f^{0}(x ; y)$, there exists a point $z \equiv T\left(h_{z}, r_{z}\right) \in U$ and a $\lambda \in(0,1)$ such that $\|(z+\lambda y)-x\|<\delta,\|z-x\|<\delta$, and

$$
\frac{f(z+\lambda y)-f(z)}{\lambda}>f^{0}(x ; y)-\epsilon .
$$


Since $f$ is locally Lipschitz on $U$, we may, by possibly moving $z$ slightly, assume that $h_{z} \in H \backslash N_{H}$. Now, by the Mean-Value theorem there exists a set $M$ of positive measure in the interval $(z, z+\lambda y)$, where $f^{\prime}\left(z^{\prime} ; y\right)$ exists and

$$
f^{\prime}\left(z^{\prime} ; y\right) \geq \frac{f(z+\lambda y)-f(z)}{\lambda}>f^{0}(x ; y)-\epsilon .
$$

Choose $z_{0} \in M \backslash N \subseteq U$ (this is possible since $h_{z} \notin N_{H}$ ). Then $\left\|z_{0}-x\right\|<\delta$, and so

$$
f^{0}(x ; y)-\epsilon<f^{\prime}\left(z_{0} ; y\right) \leq \sup \left\{f^{-}(z ; y):\|z-x\|<\delta \text { and } z \in U \backslash N\right\} .
$$

This completes the proof.

We now isolate the class of essentially smooth functions. A real-valued locally Lipschitz function $f$ defined on a nonempty open subset $U$ of a Banach space $X$ is called essentially smooth on $U$ if for each $y \in X \backslash\{0\}$

$$
\left\{x \in U: f^{0}(x ; y) \neq-f^{0}(x ;-y)\right\} \text { is a Haar-null subset of } X .
$$

Furthermore, we shall denote by $\mathcal{S}_{e}(U)$ the family of all real-valued essentially smooth locally Lipschitz functions on $U$.

Our first observation concerning these functions is that on separable Banach spaces they are just those locally Lipschitz functions which are strictly differentiable almost everywhere. (Note that in $[2,6,19]$ such functions were called essentially strictly differentiable.)

THEOREM 3.3. Let $U$ be a nonempty open subset of a separable Banach space $X$. Then each member of $\mathcal{S}_{e}(U)$ is strictly differentiable almost everywhere in $U$.

Proof. Let $\left\{y_{n}: n \in N\right\}$ be a dense subset of $X \backslash\{0\}$. For each $n \in N$, let $N_{n} \equiv\left\{x \in U: f^{0}\left(x, y_{n}\right) \neq-f^{0}\left(x ;-y_{n}\right)\right\}$. We claim that $f$ is strictly differentiable at each point of $U \backslash \bigcup\left\{N_{n}: n \in N\right\}$. To see this, consider a fixed point $x_{0} \in U \backslash \bigcup\left\{N_{n}\right.$ : $n \in N\}$. Now, as both mappings $y \rightarrow f^{0}\left(x_{0} ; y\right)$ and $y \rightarrow-f^{0}\left(x_{0} ;-y\right)$ are continuous on $X$ and $f^{0}\left(x_{0} ; y_{n}\right)=-f^{0}\left(x_{0} ;-y_{n}\right)$ for each $n \in N$, we must have that $f^{0}\left(x_{0} ; y\right)=$ $-f^{0}\left(x_{0} ;-y\right)$ for each $y \in X$. This shows that $f$ is strictly differentiable at $x_{0}$ and, hence, almost everywhere in $U$.

In order to establish that the essentially smooth functions are $D$-representable (see below) we shall need some further definitions.

A set-valued mapping $\Phi$ from a topological space $U$ into subsets of a linear topological space $(X, \tau)$ is called a $\tau$-cusco on $U$ if

(i) for each $x \in U, \Phi(x)$ is nonempty, convex, and compact;

(ii) for each open subset $W$ of $X,\{x \in U: \Phi(x) \subseteq W\}$ is open in $U$.

(Set-valued mappings satisfying only (ii) are called upper or outer semicontinuous.)

Our interest in cusco mappings arises from the fact that the Clarke subdifferential mapping of a locally Lipschitz function defined on a nonempty open subset of a Banach space is, a fortiori, a weak* cusco. Amongst the class of cusco mappings, special attention is given to the class of minimal cuscos. A cusco mapping $\Phi$ from a topological space $U$ into subsets of a linear topological space $(X, \tau)$ is called a minimal $\tau$-cusco if its graph does not strictly contain the graph of any other $\tau$-cusco defined on $U$.

We may now present the following central theorem.

THEOREM 3.4. Let $U$ be a nonempty open subset of a Banach space $X$. Then the Clarke subdifferential mapping of each member of $\mathcal{S}_{e}(U)$ is a minimal weak ${ }^{*}$ cusco on $U$. 
Proof. Let $f \in \mathcal{S}_{e}(U)$ and let us suppose, for the purpose of obtaining a contradiction, that $x \rightarrow \partial f(x)$ is not a minimal weak* cusco on $U$. That is, let us suppose that there exists a weak* cusco $\Phi$ on $U$ whose graph is strictly contained in that of $\partial f$. It follows then, via a separation argument in $\left(X^{*}\right.$, weak $\left.k^{*}\right)$, that we may find a point $x_{0} \in U$ and a direction $\hat{y} \in \hat{X}$ (here $\hat{X}$ denotes the natural embedding of $X$ into $\left.X^{* *}\right)$ such that

$$
\max \hat{y}\left(\Phi\left(x_{0}\right)\right)<\max \hat{y}\left(\partial f\left(x_{0}\right)\right) .
$$

Now consider the two cusco mappings $T_{y}: U \rightarrow 2^{R}$ and $S_{y}: U \rightarrow 2^{R}$ defined by

$$
S_{y}(x) \equiv \hat{y}(\Phi(x)) \text { and } T_{y}(x) \equiv \hat{y}(\partial f(x)) .
$$

Clearly, $S_{y}(x) \subseteq T_{y}(x)$ for each $x \in U$. However, $S_{y}\left(x_{0}\right) \neq T_{y}\left(x_{0}\right)$ since

$$
\max S_{y}\left(x_{0}\right)=\max \hat{y}\left(\Phi\left(x_{0}\right)\right)<\max \hat{y}\left(\partial f\left(x_{0}\right)\right)=\max T_{y}\left(x_{0}\right) .
$$

So we will obtain our desired contradiction if we can show that $T_{y}=S_{y}$. Let $N \equiv$ $\left\{x \in U: f^{0}(x ; y) \neq-f^{0}(x ;-y)\right\}$. It is clear then, from the definition of $T_{y}$, that

$$
S_{y}(x)=T_{y}(x)=\left[-f^{0}(x ;-y), f^{0}(x ; y)\right]=\left\{f^{\prime}(x ; y)\right\} \text { at each point of } U \backslash N .
$$

We may now use the upper semicontinuity of $S_{y}$, a simple separation argument (in $R$ ), and Theorem 3.2 to deduce that $S_{y}=T_{y}$.

The importance of the previous result is that minimality of the Clarke subdifferential mapping is closely related to the, about to be defined, notion of $D$-representability. Moreover, minimality of the Clarke subdifferential mapping also provides a means for deducing differentiability results. Indeed, let us recall that a Banach space $X$ is said to be of $\operatorname{class}(\mathcal{S})$ if every minimal weak* cusco that maps from a Baire space, into subsets of $X^{*}$, is single valued at the points of a dense and $G_{\delta}$ subset of its domain (see [10]). Consequently, we see that in these spaces the essentially smooth functions are strictly differentiable at the points of a dense and $G_{\delta}$ subset of their domain. The family of all class $(\mathcal{S})$ spaces is rather large. Indeed, all smooth Banach spaces (that is, spaces which admit an equivalent norm that is Gâteaux differentiable everywhere except at 0 ) belong to this class (see [10]), as do all those Banach spaces that contain, as a dense subspace, the continuous linear image of an Asplund space (such spaces are called $G S G$ spaces [10]).

Let $f$ be a real-valued locally Lipschitz function defined on a nonempty open subset $U$ of a $\operatorname{class}(\mathcal{S})$ Banach space $X$. Then we say that $f$ is $D$-representable on $U$ if $D \equiv\{x \in U: \nabla f(x)$ exists $\}$ is dense in $U$ and for each dense subset $D^{*}$ of $D$,

$$
\partial f(x)=\overline{c o}^{w^{*}}\left\{x^{*} \in X^{*}: x^{*}=w_{e a k^{*}-} \lim _{\substack{x_{n} \rightarrow x \\ x_{n} \in D^{*}}} \nabla f\left(x_{n}\right)\right\} .
$$

The next theorem now follows directly from Corollary 2.2 and Theorem 2.5 in [4].

THEOREM 3.5. Let $U$ be a nonempty open subset of a class $(\mathcal{S})$ Banach space. Then each member of $\mathcal{S}_{e}(U)$ is D-representable.

The next property that we shall address is that of integrability. Let $f$ be a realvalued locally Lipschitz function defined on a nonempty open subset $U$ of a Banach space $X$. Then we say that $f$ is integrable on $U$ if $\partial(f-g) \equiv\{0\}$ for each real-valued locally Lipschitz function $g$, defined on $U$, with $\partial g(x) \subseteq \partial f(x)$ for each $x \in U$.

We now show that the essentially smooth functions are integrable. 
Proposition 3.6. Suppose that $U$ is a nonempty open subset of a Banach space $X$. Let $f$ and $g$ be real-valued locally Lipschitz functions on $U$ such that $\partial g(x) \subseteq \partial f(x)$ for each $x \in U$. Then $\partial(f-g) \equiv\{0\}$ if and only if $x \rightarrow \partial(f-g)(x)$ is a minimal weak* cusco on $U$.

Proof. Since $f=(f-g)+g$ we have that $\partial f(x) \subseteq \partial(f-g)(x)+\partial g(x) \subseteq$ $\partial(f-g)(x)+\partial f(x)$ and "cancellation" of closed bounded convex sets implies that $0 \in \partial(f-g)(x)$ for all $x \in U$. So if $x \rightarrow \partial(f-g)(x)$ is a minimal weak ${ }^{*}$ cusco on $U$, then $\partial(f-g) \equiv\{0\}$. The converse is obvious.

Corollary 3.7. Suppose that $U$ is a nonempty open connected subset of a Banach space $X$. Let $f$ and $g$ be real-valued locally Lipschitz functions defined on $U$ such that $\partial g(x) \subseteq \partial f(x)$ for each $x \in U$. Then $f-g$ is constant on $U$ if and only if $x \rightarrow \partial(f-g)(x)$ is a minimal weak* cusco on $U$.

Proof. Suppose that $x \rightarrow \partial(f-g)(x)$ is a minimal weak* cusco on $U$. Let $x_{0} \in U$, and let $U_{0} \equiv\left\{x \in U:(f-g)(x)=(f-g)\left(x_{0}\right)\right\}$. It follows from the Mean-Value theorem and the result above that both $U_{0}$ and $U \backslash U_{0}$ are open subsets of $U$. Now, $U_{0} \neq \emptyset$ since $x_{0} \in U_{0}$. Therefore, $U_{0}=U$, which shows that $f-g$ is constant on $U$. The converse is again obvious.

THEOREM 3.8. Let $U$ be a nonempty open subset of a Banach space $X$. Then each member of $\mathcal{S}_{e}(U)$ is integrable.

Proof. Suppose that $f \in \mathcal{S}_{e}(U)$ and $g$ is any real-valued locally Lipschitz function defined on $U$ such that $\partial g(x) \subseteq \partial f(x)$ for all $x \in U$. Clearly then $g \in \mathcal{S}_{e}(U)$. Moreover, since $\partial(f-g)(x) \subseteq \partial f(x)-\partial g(x)$ we see that $f-g \in \mathcal{S}_{e}(U)$, and so the subdifferential mapping, $x \rightarrow \partial(f-g)(x)$, is a minimal weak* cusco on $U$. Hence, by the previous Proposition, $\partial(f-g) \equiv\{0\}$ on $U$.

We say that a locally Lipschitz function $f$ is hereditarily integrable on an open subset $U$ if its restriction to every open subset is integrable (on that open set). Hence, we see that the members of $\mathcal{S}_{e}(U)$ are not only integrable, but also hereditarily integrable on $U$. It is appropriate to recall that there are Lipschitz functions which are integrable but not hereditarily integrable (see [4, section 7$]$ ).

THEOREM 3.9 (identity theorem). Suppose that $f$ and $g$ are real-valued locally Lipschitz functions defined on a nonempty open connected subset $U$ of a Banach space $X$. If $f \in \mathcal{S}_{e}(U)$ and $x \rightarrow \partial g(x)$ is a minimal weak* cusco, then $f-g \equiv$ constant on $U$ if and only if $\{x \in U: \partial g(x) \cap \partial f(x) \neq \emptyset\}$ is dense in $U$.

Proof. Consider the set-valued mapping $T: U \rightarrow 2^{X^{*}}$ defined by $T(x) \equiv \partial g(x) \cap$ $\partial f(x)$. Since both mappings, $x \rightarrow \partial f(x)$ and $x \rightarrow \partial g(x)$, are upper semicontinuous on $U$ (and possess compact images) $T$ possesses nonempty weak* compact convex images. Moreover, since the graphs of both $\partial f$ and $\partial g$ are closed in $U \times X^{*}$, with $X^{*}$ equipped with the weak* topology, so is the graph of $T$. Hence, we may deduce that $T$ is a cusco on $U$. However, for each $x \in U, T(x) \subseteq \partial f(x)$ and $T(x) \subseteq \partial g(x)$. Therefore, by the minimality of $\partial f$ and $\partial g$ we must have that $\partial g=T=\partial f$. The result now follows from Theorem 3.8. Yet again, the converse is obvious.

In the following theorem we provide a condition which is sufficient to ensure membership in $\mathcal{S}_{e}(U)$.

THEOREM 3.10. Let $f$ be a real-valued locally Lipschitz function defined on a nonempty open subset $U$ of a Banach space $X$. Let $B$ be any subset of $X$ such that $\overline{s p} B=X$. If for each $b \in B,\left\{x \in U: f^{0}(x ; b) \neq-f^{0}(x ;-b)\right\}$ is a Haar-null set, then $f \in \mathcal{S}_{e}(U)$.

Proof. Let $y \in X \backslash\{0\}$. We will show that $\left\{x \in U: f^{0}(x ; y) \neq-f^{0}(x ;-y)\right\}$ is a Haar-null set. We may choose a countable set $B^{\prime} \equiv\left\{b_{n}: n \in N\right\} \subseteq B$ so that 
$y \in \overline{s p} B^{\prime}$. For each $n \in N$, let $S_{n} \equiv\left\{x \in U: f^{0}\left(x ; b_{n}\right)=-f^{0}\left(x ;-b_{n}\right)\right\}$. It is clear from the hypothesis that each set $U \backslash S_{n}$ is Haar-null. Let $S \equiv \bigcap\left\{S_{n}: n \in N\right\}$. We claim that $f$ is strictly differentiable, in the direction $y$, at each point of $S$. So let us consider an arbitrary point $x_{0} \in S$. To show that $f$ is strictly differentiable at $x_{0}$, in the direction $y$, we need only show that if $x_{1}^{*}$ and $x_{2}^{*}$ are Clarke subgradients of $f$ at $x_{0}$, then $x_{1}^{*}(y)=x_{2}^{*}(y)$ (this is because, if $f^{0}\left(x_{0} ; y\right) \neq-f^{0}\left(x_{0} ;-y\right)$, then we could construct, using the Hahn-Banach extension theorem, two distinct subgradients $x_{1}^{*}$ and $x_{2}^{*}$ of $f$, at $x_{0}$, such that $x_{1}^{*}(y)=f^{0}\left(x_{0} ; y\right)$ and $\left.x_{2}^{*}(y)=-f^{0}\left(x_{0} ;-y\right)\right)$. To this end, let $x_{1}^{*}$ and $x_{2}^{*}$ be Clarke subgradients of $f$ at $x_{0}$. Then, $x_{i}^{*}\left(b_{n}\right) \leq f^{0}\left(x_{0} ; b_{n}\right)$ and $x_{i}^{*}\left(-b_{n}\right) \leq f^{0}\left(x_{0} ;-b_{n}\right)$ for each $i \in\{1,2\}$ and $n \in N$. Therefore,

$$
x_{i}^{*}\left(b_{n}\right) \leq f^{0}\left(x_{0} ; b_{n}\right)=-f^{0}\left(x_{0} ;-b_{n}\right) \leq-x_{i}^{*}\left(-b_{n}\right)=x_{i}^{*}\left(b_{n}\right)
$$

for each $i \in\{1,2\}$ and $n \in N$. Hence, $x_{1}^{*}\left(b_{n}\right)=f^{0}\left(x_{0} ; b_{n}\right)=x_{2}^{*}\left(b_{n}\right)$ for each $n \in N$. Now, both $x_{1}^{*}$ and $x_{2}^{*}$ are linear on $X$, and so we have that $x_{1}^{*}(b)=x_{2}^{*}(b)$ for each $b \in s p B^{\prime}$. However, $x_{1}^{*}$ and $x_{2}^{*}$ are also both continuous on $X$; therefore, $x_{1}^{*}=x_{2}^{*}$ on $\overline{s p} B^{\prime}$. In particular, $x_{1}^{*}(y)=x_{2}^{*}(y)$.

Let $f$ be a real-valued locally Lipschitz function defined on a nonempty open subset $U$ of a Banach space $X$. Then $f$ is upper hemismooth (lower hemismooth) at a point $x \in U$, in the direction $y$, if

$$
f^{+}(x ; y) \geq \limsup _{t \rightarrow 0^{+}} f^{0}(x+t y ; y) \quad\left(f^{-}(x ; y) \leq \liminf _{t \rightarrow 0^{+}}-f^{0}(x+t y ;-y)\right) .
$$

If $f$ is both upper hemismooth and lower hemismooth at $x \in U$, in the direction $y$, then we say that $f$ is hemismooth at $x$, in the direction $y$.

Remark 3.1. If we define

$$
T_{y}: U \rightarrow R \text { by, } T_{y}(x) \equiv \limsup _{t \rightarrow 0^{+}} f^{0}(x+t y ; y)
$$

and

$$
S_{y}: U \rightarrow R \text { by, } S_{y}(x) \equiv \liminf _{t \rightarrow 0^{+}}-f^{0}(x+t y ;-y),
$$

then it is easy to check that both $T_{y}$ and $S_{y}$ are Borel measurable on $U$. Hence, the set of points in $U$ where $f$ is upper (lower) hemismooth, in the direction $y$, is always a Borel subset of $U$. Indeed, to see that $T_{y}$ is Borel measurable, it suffices to observe that

$$
T_{y}(x)=\lim _{n \rightarrow \infty} g_{n}(x), \text { where } g_{n}(x) \equiv \sup \left\{f^{0}(x+t y ; y): 0<t \leq 1 / n\right\},
$$

and

$$
g_{n}(x) \equiv \lim _{m \rightarrow \infty} f_{m}^{n}(x), \text { where } f_{m}^{n}(x) \equiv \max \left\{f^{0}(x+t y ; y): 1 / m \leq t \leq 1 / n\right\}
$$

(for each $m>n$ ), are upper semicontinuous on $U$. A similar argument shows that $S_{y}$ is also Borel measurable.

Further, we say that $f$ is essentially upper hemismooth (essentially lower hemismooth) on $U$, in the direction $y$, if the set of all points in $U$ where $f$ is not upper hemismooth (lower hemismooth), in the direction $y$, is a Haar-null set. We shall also say that $f$ is pseudoregular at $x$, in the direction $y$, if $f^{0}(x ; y)=f^{+}(x ; y)$, and we shall say that $f$ is pseudoregular at $x$ if it is pseudoregular at $x$ in every direction $y$. 
LEMMA 3.11. Let $f$ be a real-valued locally Lipschitz function defined on a nonempty open subset $U$ of a Banach space $X$. Then, in the notation of the previous remark, for each $y \in S(X)$ the (Borel) set

$$
F_{y} \equiv\left\{x \in U: f^{0}(x ; y)>T_{y}(x)\right\} \quad\left(E_{y} \equiv\left\{x \in U:-f^{0}(x ;-y)<S_{y}(x)\right\}\right)
$$

has the property that for each $x_{0} \in U, F_{y}\left(x_{0}\right) \equiv\left\{r \in R: x_{0}+r y \in F_{y}\right\}\left(E_{y}\left(x_{0}\right) \equiv\right.$ $\left.\left\{r \in R: x_{0}+r y \in E_{y}\right\}\right)$ is at most countable.

Proof. Fix $y \in S(X)$ and $x_{0} \in U$. We will show that $F_{y}\left(x_{0}\right)$ is at most countable (the proof that $E_{y}\left(x_{0}\right)$ is countable is identical to this). Note that without loss of generality we may assume that $F_{y}\left(x_{0}\right)$ is nonempty. So in this case, we define $s: F_{y}\left(x_{0}\right) \rightarrow \mathbf{Q}^{2}$ by $s(t) \equiv\left(r_{1}, r_{2}\right)$, where $r_{1} \in\left(T_{y}\left(x_{0}+t y\right), f^{0}\left(x_{0}+t y ; y\right)\right) \cap \mathbf{Q}$ and $r_{2} \in(t, \infty) \cap \mathbf{Q}$ is chosen so that

$$
\sup \left\{f^{0}\left(x_{0}+r y ; y\right): t<r<r_{2}\right\}<r_{1} .
$$

It is easy to see that $s$ is 1 to 1 , and so $F_{y}$ must be at most countable. Indeed, if $t_{1}<t_{2}$ and $s\left(t_{1}\right)=s\left(t_{2}\right)=\left(r_{1}, r_{2}\right)$, then $t_{1}<t_{2}<r_{2}$ and so $f^{0}\left(x_{0}+t_{2} y ; y\right)<r_{1}$. However, this is impossible since it contradicts the definition of $s$ at $t_{2}$. (Here, $\mathbf{Q}$ denotes the rational numbers.)

Remark 3.2. If $X$ is a Banach space, then for each $y \in S(X),\{x \in U$ : $f$ is upper hemismooth, but not pseudoregular, in the direction $y$ \} is contained in $F_{y}$ and hence is a Haar-null set (in this case, we may take the normalized Lebesgue measure, supported on $\operatorname{sp}\{y\}$, as a test measure for the Borel set $F_{y}$ ).

LEMMA 3.12. Let $f$ be a real-valued locally Lipschitz function defined on a nonempty open subset $U$ of a Banach space $X$. If for some $y \in X \backslash\{0\} f$ is essentially upper hemismooth (essentially lower hemismooth) in the direction $y$, then $f^{0}(x ; y)=f^{\prime}(x ; y)\left(-f^{0}(x ;-y)=f^{\prime}(x ; y)\right)$ almost everywhere in $U$.

Proof. Suppose that $f$ is essentially upper hemismooth in the direction $y$ (the proof for the case when $f$ is essentially lower hemismooth in the direction $y$ is similar). It follows from Remark 3.2 that $f^{0}(x ; y)=f^{+}(x ; y)$ almost everywhere in $U$. It now only remains to observe that $f^{+}(x ; y)=f^{\prime}(x ; y)$ almost everywhere in $U$ (to see this, take the normalized Lebesgue measure, supported on $s p\{y\}$, as a test measure for the set $\left\{x \in U: f^{\prime}(x ; y)\right.$ does not exist $\left.\}\right)$.

Now from Lemma 3.12 and Theorem 3.10 we may deduce the following theorem.

THEOREM 3.13. Let $f$ be a real-valued locally Lipschitz function defined on a nonempty open subset $U$ of a Banach space $X$. Let $B$ be any subset of $X$ such that $\overline{s p} B=X$. If for each $b \in B$ either, (i) $f$ is essentially upper hemismooth in the directions $b$ and $-b$; (ii) $f$ is essentially lower hemismooth in the directions $b$ and $-b$; (iii) $f$ is essentially hemismooth in the direction $b$; or, (iv) $f$ is essentially hemismooth in the direction $-b$, then $f \in \mathcal{S}_{e}(U)$.

It follows from the above theorem that the class of essentially smooth functions is quite large, and as we shall see next, also quite robust. More precisely, we will show that the essentially smooth functions are closed under addition, subtraction, multiplication, and division (when this is defined), as well as the lattice operations. Actually, it not unreasonable to expect that the essentially smooth functions obey closure properties considerably stronger than those just mentioned. In fact, a first, but naive, guess might be that if $f_{1}, f_{2}, \ldots, f_{n} \in \mathcal{S}_{e}(U)$ and $g \in \mathcal{S}_{e}\left(R^{n}\right)$, then $g \circ f \in \mathcal{S}_{e}(U)$, where $f \equiv\left(f_{1}, f_{2}, \ldots, f_{n}\right)$. To the contrary, in [5], the authors give an example that shows that this is not true when $n \geq 2$. 
For the final result of the paper we will need to consider vector-valued functions. Let $U$ be a nonempty open subset of a Banach space $X$, and let $V$ be a nonempty open subset of $R^{n}$. If $x: U \rightarrow V$ is defined by

$$
x(t) \equiv\left(x_{1}(t), x_{2}(t), \ldots, x_{n}(t)\right) \quad \text { with } x_{j}: U \rightarrow R,
$$

then we say that the vector-valued function $x$ is essentially smooth on $U$ if $x_{j} \in \mathcal{S}_{e}(U)$ for each $1 \leq j \leq n$ and in this case we write $x \in \mathcal{S}_{e}(U ; V)$. In addition to this, we will say that a real-valued locally Lipschitz function $f$ defined on a nonempty open subset $V$ on $R^{n}$ is arc-wise essentially smooth on $V$, if for each locally Lipschitz function $x \in \mathcal{S}_{e}((0,1) ; V)$

$$
\lambda\left(\left\{t \in(0,1): f^{0}\left(x(t) ; x^{\prime}(t)\right) \neq-f^{0}\left(x(t) ;-x^{\prime}(t)\right)\right\}\right)=0 .
$$

$$
\text { [Here } x^{\prime}(t) \equiv\left(x_{1}^{\prime}(t), x_{2}^{\prime}(t), \ldots, x_{n}^{\prime}(t)\right) \text {.] }
$$

We shall denote by $\mathcal{A}_{e}(V)$ the family of all arc-wise essentially smooth Lipschitz functions on $V$. A relevant fact for the next theorem is that $\mathcal{A}_{e}(V)$ contains all the pseudoregular and semismooth (see [18] for definition) functions on $V$. In particular, $\mathcal{A}_{e}(V)$ contains all the continuous convex and $C^{1}$ functions on $V$ (see [5]).

THEOREM 3.14 (see [5, Theorem 2.3]). Let $U$ be a nonempty open subset of a Banach space $X$, and let $f \equiv\left(f_{1}, f_{2}, \ldots, f_{n}\right) \in \mathcal{S}_{e}(U ; V)$, where $V$ is any nonempty open subset of $R^{n}$ that contains $f(U)$. Then $g \circ f \in \mathcal{S}_{e}(U)$ whenever $g \in \mathcal{A}_{e}(V)$.

Remark 3.3. In [4] it is shown that if $U$ is a nonempty open subset of $R$, then $\mathcal{S}_{e}(U)=\mathcal{A}_{e}(U)$. However, it is also shown in [4] that if $U$ is a nonempty open subset of $R^{n}(n \geq 2)$, then $\mathcal{A}_{e}(U)$ is a proper subset of $\mathcal{S}_{e}(U)$.

\section{REFERENCES}

[1] C. Berg, J. P. R. Christensen, And P. Ressel, Harmonic Analysis on Semigroups, SpringerVerlag, New York, 1984.

[2] J. M. Borwein, Minimal cuscos and subgradients of Lipschitz functions, in Fixed Point Theory and its Applications, J.-B. Baillion and M. Thera, eds., Pitman Lecture Notes in Math., Longman Press, London, 1991, pp. 57-82.

[3] J. M. Borwein And S. P. Fitzpatrick, Closed convex Haar null sets, CECM report 95:052, Simon Fraser University, Burnaby, BC, Canada.

[4] J. M. Borwein And W. B. Moors, Essentially smooth Lipschitz functions, J. Funct. Anal., 149 (1997), pp. 305-351.

[5] J. M. Borwein AND W. B. Moors, A chain rule for essentially smooth Lipschitz functions, SIAM J. Optim., 8 (1998), pp. 300-307.

[6] J. M. BorWein AND W. B. MoOrs, Lipschitz functions with minimal Clarke subdifferential mappings, in Proc. Optim. Miniconference III: The University of Melbourne, July 18, 1996, B. M. Glover, B. D. Craven, and D. Ralph, eds., Ballarat, Victoria, 1997, pp. 5-12.

[7] J. P. R. Christensen, On sets of Haar measure zero in Abelian groups, Israel J. Math., 13 (1972), pp. 255-260.

[8] F. H. Clarke, Optimization and Nonsmooth Analysis, John Wiley, New York, 1983.

[9] R. M. Dudley, Real Analysis and Probability, Wadsworth and Brooks/Cole Math. Ser., Wadsworth and Brooks/Cole Software, Pacific Grove, CA, 1989.

[10] M. Fabian, Gateaux Differentiability of Convex Functions and Topology-Weak Asplund Spaces, John Wiley and Sons, Inc., Adv. Books, New York, 1997.

[11] B. R. Hunt, T. Sauer, And J. A. Yorke, Prevalence: A translation-invariant "almost every" on infinite-dimensional spaces, Bull. Amer. Math. Soc., 27 (1992), pp. 217-238.

[12] J. E. Jayne, I. Namioka, And C. A. Rogers, Topological properties of Banach spaces, Proc. London Math. Soc. (3), 66 (1993), pp. 651-672.

[13] A. S. Kechris, Topology and descriptive set theory, Topology Appl., 58 (1994), pp. 195-222. 
[14] V. L. KLEE, Invariant metrics in groups (Solution of a problem of Banach), Proc. Amer. Math. Soc, 3 (1952), pp. 484-487.

[15] P. MANKIEwicz, On the differentiability of Lipschitz mappings in Frechet spaces, Studia Math., 45 (1973), pp. 15-29.

[16] E. Matouskova, Convexity and Haar null sets, Proc. Amer. Math. Soc., 125 (1997), pp. 17931799.

[17] E. Matouskova And C. Stegall, A characterization of reflexive Banach spaces, Proc. Amer. Math. Soc., 144 (1996), pp. 1083-1090.

[18] R. Mifflin, Semismooth and semiconvex functions in optimization, SIAM J. Control Optim., 15 (1977), pp. 959-972.

[19] W. B. Moors, A characterisation of minimal subdifferential mappings of locally Lipschitz functions, Set-Valued Anal., 3 (1995), pp. 129-141.

[20] R. R. Phelps, Gaussian null sets and differentiability of Lipschitz functions on Banach spaces, Pacific J. Math., 77 (1978), pp. 523-531.

[21] E. Szpilrajn, Sur certain invariants de l'operation (A), Fund. Math., 21 (1933), pp. 229-235. 